\title{
Overdispersed gene expression in schizophrenia
}

\author{
Guangzao Huang ${ }^{1,2,7,8}$, Daniel Osorio $\mathbb{D}^{3,8}$, Jinting Guan ${ }^{1,2}$, Guoli Ji ${ }^{1,2,4 凶}$ and James J. Cai $\mathbb{D}^{3,5,6 \bowtie}$
}

Schizophrenia (SCZ) is a severe, highly heterogeneous psychiatric disorder with varied clinical presentations. The polygenic genetic architecture of SCZ makes identification of causal variants a daunting task. Gene expression analyses hold the promise of revealing connections between dysregulated transcription and underlying variants in SCZ. However, the most commonly used differential expression analysis often assumes grouped samples are from homogeneous populations and thus cannot be used to detect expression variance differences between samples. Here, we applied the test for equality of variances to normalized expression data, generated by the CommonMind Consortium (CMC), from brains of 212 SCZ and 214 unaffected control (CTL) samples. We identified 87 genes, including VEGFA (vascular endothelial growth factor) and BDNF (brain-derived neurotrophic factor), that showed a significantly higher expression variance among SCZ samples than CTL samples. In contrast, only one gene showed the opposite pattern. To extend our analysis to gene sets, we proposed a Mahalanobis distance-based test for multivariate homogeneity of group dispersions, with which we identified 110 gene sets with a significantly higher expression variability in SCZ, including sets of genes encoding phosphatidylinositol 3-kinase (PI3K) complex and several others involved in cerebellar cortex morphogenesis, neuromuscular junction development, and cerebellar Purkinje cell layer development. Taken together, our results suggest that SCZ brains are characterized by overdispersed gene expression-overall gene expression variability among SCZ samples is significantly higher than that among CTL samples. Our study showcases the application of variability-centric analyses in SCZ research.

npj Schizophrenia (2020)6:9; https://doi.org/10.1038/s41537-020-0097-5

\section{INTRODUCTION}

Schizophrenia (SCZ) - one of the most severe psychiatric disorders -affects about $1 \%$ of the general population ${ }^{1-3}$. The disorder manifests itself in many different forms and includes both positive behaviors, such as delusions, hallucinations, and disorganized speech, and negative behaviors, such as the absence of reaction, loss of interest in everyday activities, and lack of feeling or emotion. SCZ affects patients differently. People with the disorder vary widely in their symptoms, course of illness, and treatment response ${ }^{4}$. A reliable clinical typology of SCZ has proved difficult to develop ${ }^{5-7}$. Assessment of clinical outcomes in SCZ is challenging ${ }^{8,9}$. Patients diagnosed with SCZ can be classified into those with and without neurodevelopmental impairment ${ }^{10-12}$. The former category is likely to be due to the impact of risk alleles, copy number variants (CNVs), or early environmental insults such as hypoxic damage to the hippocampus. The latter is more likely to be due to affective dysregulation. Detailed neuroimaging and many measures of psychological variables can also be used to classify SCZ patients into distinct subgroups ${ }^{13-16}$. The existence of multiple ways to classify SCZ underscores the marked betweenpatient variability and within-category heterogeneity associated with the disorder. Thus, SCZ is a highly heterogeneous group of disorders rather than a single disease ${ }^{17-19}$.

Although SCZ was described more than 100 years ago, the exact etiology and genetic mechanism of SCZ are still unclear. With an upper bound estimate of heritability of $80 \%{ }^{20}$, the risk of SCZ is clearly under the substantial genetic influence. Numerous common single nucleotide polymorphisms (SNPs) (refs ${ }^{21-23}$ ) and CNVs (refs ${ }^{24-27}$ ) have been identified to be associated with the SCZ risk. Nevertheless, like many other complex diseases, SCZ has a polygenic architecture $21,22,28$, and is influenced by environmental factors ${ }^{16}$, making it difficult to pinpoint causal mutations.

Gene expression data of SCZ patients has been collected and integrated into analyses ${ }^{29-33}$, in order to improve mechanistic interpretations of risk alleles, or directly identify dysregulated genes in relevant tissues. Most studies of SCZ transcriptome adopt the method of differential expression (DE) aimed at the identification of genes expressed significantly differently in SCZ patients than unaffected controls (CTL). However, given the fact that SCZ is highly heterogeneous, we argue that the DE analysis may not be sufficient as it treats SCZ patients as a homogeneous group of individuals, assuming gene expression in all SCZ samples, compared with that in all CTL samples, is consistently up- or downregulated. Indeed, a robust overlap between sets of DE genes identified in different SCZ transcriptome studies has been rarely observed. Many of statistically significant DE genes cannot be individually connected with any of the current pathophysiological hypotheses of the disease either.

Here, we consider a complementary alternative. We assume disease-relevant genes are expressed more variably in SCZ patients than CTL individuals and therefore lead to an 'overdispersion' in gene expression in the SCZ group. The rationale behind our argument is that patients affected with SCZ are a heterogeneous group of individuals: it is not simply that they share few or no symptoms in common ${ }^{34}$; rather, etiologically, every patient with SCZ is 'ill' in his or her own way. This can be summarized into the 'Anna Karenina principle' for SCZ, in which gene expression of affected individuals varies more than that of healthy individuals, matching with Leo Tolstoy's dictum that 'happy families are all alike; each unhappy family is unhappy in its

\footnotetext{
${ }^{1}$ Department of Automation, Xiamen University, Xiamen 361005, China. ${ }^{2}$ National Institute for Data Science in Health and Medicine, Xiamen University, Xiamen 361005, China. ${ }^{3}$ Department of Veterinary Integrative Biosciences, Texas A\&M University, College Station, TX 77843, USA. ${ }^{4}$ Innovation Center for Cell Signaling Network, Xiamen University, Xiamen 361005, China. ${ }^{5}$ Department of Electrical and Computer Engineering, Texas A\&M University, College Station, TX 77843, USA. ${ }^{6}$ Interdisciplinary Program of Genetics, Texas A\&M University, College Station, TX 77843, USA. ${ }^{7}$ Present address: College of Electrical and Electronic Engineering, Wenzhou University, Wenzhou 325035 , China. ${ }^{8}$ These authors contributed equally: Guangzao Huang, Daniel Osorio. ${ }^{凶}$ email: glji@xmu.edu.cn; jcai@tamu.edu
} 
own way'. We set out to test the hypothesis that overdispersed gene expression in SCZ is a common and important consequence of transcriptional dysregulation. The phenomenon should be more pronounced for genes and pathways that underlie SCZ pathogenesis. The pattern is easily missed or discarded by common workflows such as those implemented in the DE analysis.

The structure of this paper is as follows. First, we represent the results of our differential variability (DV) analysis on single genes, showing an overwhelming pattern of increased variability at the single-gene level associated with SCZ. Second, we introduce a newly developed, multivariate DV analysis method. Through the application of this method, we show that multiple gene sets, whose enriched functions are SCZ related, have higher expression variability among the SCZ subjects. Third, we show the contribution of common genetic variants to expression variability. To the best of our knowledge, this kind of variability-centric analysis has not been done with data sets from the SCZ cohort. We conclude by providing the interpretation of our results in the context of gene discovery and implications in the personalized intervention of SCZ.

\section{RESULTS}

Single genes with higher expression variability among SCZ samples

We obtained the normalized gene expression data from the CommonMind Consortium (CMC) study ${ }^{29}$. The data were generated using RNA sequencing (RNA-seq) from the dorsolateral prefrontal cortex of individuals affected with SCZ and unaffected control $(C T L)$ individuals. Our analyses were done with data from 212 SCZ and 214 CTL individuals, all with European ancestry. For each gene, we used the Brown-Forsythe (B-F) test (ref. ${ }^{35}$ ) to determine whether there is a significant difference in group variances between SCZ and CTL. At the level of $5 \%$ false discovery rate (FDR), we identified 88 differentially variable (DV) genes (Supplementary Table 1), including 87 with a greater expression variance in SCZ than CTL and one (TAMM41) with a smaller expression variance in SCZ than CTL. Thus, at the single-gene level, greater between-individual expression variability is an overwhelming feature of SCZ. Illustrative examples, showing DV genes' expression levels across CTL and SCZ samples, are given in Supplementary Fig. 1. The overdispersed expression in SCZ is robust against outlying samples-i.e., the pattern was not driven by outlying individuals with extreme expression values in a large number of genes. Using one of DV genes, ZBTB24, as an example, we intentionally removed two SCZ samples with the highest and lowest expression value, respectively. Our purpose was to assess the impact of these two outliers on the significance of the statistical test for the DV gene detection. The removal of these two extreme samples had no qualitative influence on the outcome of the B-F test (Supplementary Fig. 2). These results suggest that increased gene expression variability in SCZ is unlikely to be due to the existence of a small number of SCZ samples with extremely high or low expression levels. Instead, it is due to a systematic overdispersion of gene expression among SCZ samples.

Furthermore, using a data set from the BrainSeq project ${ }^{36}$, which contains the patients' information on the use of medicine, we estimated the contributions of antipsychotics and antidepressants to gene expression variability in SCZ samples. First, we identified DV genes expressed more variably in SCZ than CTL. Then, for each of these DV genes, we compared its expression variance in SCZ patients received antipsychotics treatment with that in SCZ patients not received antipsychotics treatment. No gene was found to be significant using the B-F test with the multiple test correction. Finally, we also compared the distribution of $\mathrm{B}-\mathrm{F}$ test $p$-values and the distribution obtained using randomly shuffled data. No difference was revealed either (see Supplementary Fig. 3 for QQ plots). We also plotted the expression levels of selected DV genes to visualize their distribution pattern in treated and untreated SCZ patients (Supplementary Fig. 4). Taken together, these analyses suggest that the contribution of the use of antipsychotics and antidepressant to gene expression variability in SCZ seems minimal.

Using the gene ontology (GO) enrichment test ${ }^{37}$, no significantly enriched function was found to associated with the $88 \mathrm{DV}$ genes, suggesting marked functional diversity of these genes. One of the most salient examples is VEGFA, a gene encoding vascular endothelial growth factor (Fig. 1a). This signal protein, VEGFA, stimulates vasculogenesis and angiogenesis ${ }^{38}$, playing an important role in neurogenesis, neuronal differentiation, and neuroprotection and regeneration of central nervous system (CNS) cells ${ }^{39-41}$. Data from an independent microarray-based study confirms the a

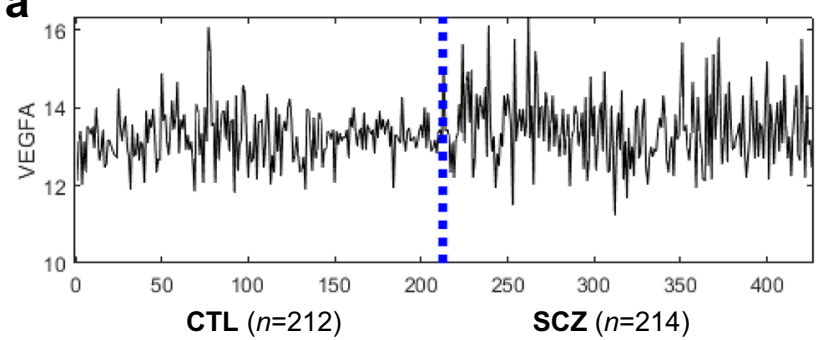

b

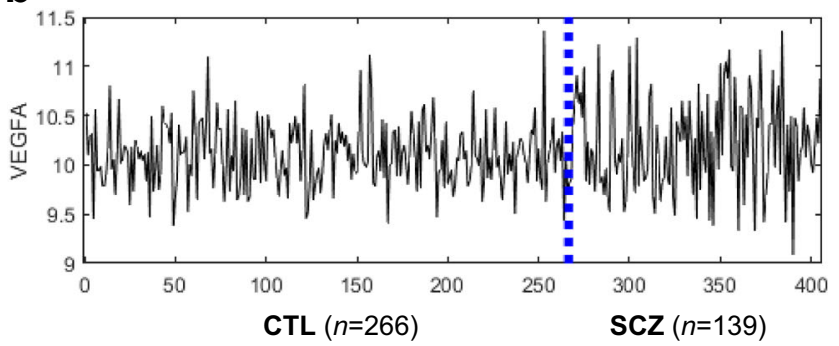

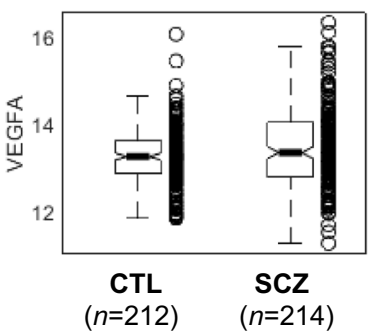

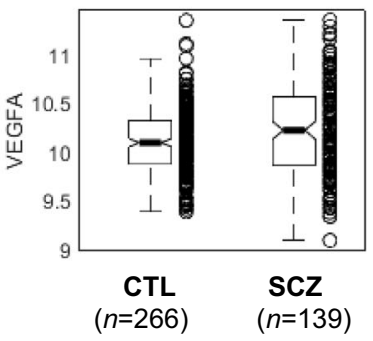

Fig. 1 Expression profiles of VEGFA show more dispersed expression in SCZ than CTL. a Normalized expression values in 212 CTL and 214 SCZ samples generated by the CMC study. B-F test $p$-value $=3.6 \mathrm{e}-6$. b Normalized expression values in 266 CTL and 139 SCZ samples, as reported in ref. ${ }^{42}$. B-F test $P$-value $=1.7 \mathrm{e}-9$. 
higher VEGFA expression variability in SCZ samples (Fig. 1b) ${ }^{42}$. In addition to VEGFA, BDNF is a member of the neurotrophin family of growth factors related to the canonical nerve growth factor; NECTIN2 encodes a single-pass type I membrane glycoprotein implicated in Alzheimer's disease; AIF1 encodes allograft inflammatory factor found in activated macrophages in tissues with inflammation (Supplementary Fig. 1). Furthermore, HPS5, STAR, TMEM125, RPN2, and DNAH1 loci are known to contain genetic variants associated with $\mathrm{SCZ}^{43-47}$.

Gene sets with higher expression variability among SCZ samples Next, we turned our focus from single genes to gene sets. To extend our analysis to gene sets, we adapted the procedure for analyzing multivariate homogeneity of group dispersions ${ }^{48}$. This procedure is a multivariate analog of Levene's test for homogeneity of variances and has been widely used in ecology, for example, in defining the variability in species composition. In the proposed method, we used Mahalanobis distance (MD) to replace Euclidian distance originally used in the procedure. This adaptation is essential to account for collinearity in expression levels of genes in each gene set across samples ${ }^{49,50}$. To increase the power of the test, we introduced further modification in our MD-based test (see Methods for details). We applied this modified version to gene sets, which are pre-defined using GO terms in ontologies of biological process and molecular function. We identified 110 gene sets with greater multivariate expression variance in SCZ, all with a nominal $p$-value $<0.05$ (Supplementary Table 2).

The top 30 most significant gene sets are given in Table 1. The most significant gene set contains 15 out of 20 genes involved in forming the phosphatidylinositol 3-kinase (PI3K) complex. The PI3K signaling pathway is known to be implicated in $\mathrm{SCZ}^{51-54}$. Among the rest of gene sets, many are related to brain development such as (1) cerebellar cortex morphogenesis, (2) cerebellar cortex formation, (3) neuromuscular junction development, (4) cerebellar Purkinje cell layer development, (5) cell differentiation in hindbrain, and (6) regulation of astrocyte differentiation, suggesting that $\mathrm{SCZ}$ brains are characterized by abnormally high expression variance in genes involved in the brain development. This finding is consistent with the general consensus that SCZ is a brain disorder with structural and functional changes in the cortex and the connections between different cortical regions ${ }^{11,16,55}$. In addition to these gene sets pertaining to brain development, several other gene sets are likely to be implicated in SCZ. These include necrotic cell death ${ }^{56,57}$, regulation of endoplasmic reticulum unfolded protein response $e^{58}$, and folic acid-containing compound metabolic process $^{59-61}$. For each significant gene set, we use the method of Garthwaite-Koch partition to estimate the relative contribution of each gene in the gene set to $M D^{62}$. We present the top five genes that contribute most to the MD for each gene set in Table 1 .

To illustrate the overdispersion pattern of gene set expression in $\mathrm{SCZ}$, we used the gene set of cerebellar cortex morphogenesis as

Table 1. Functional gene sets showing higher expression variability in SCZ compared with CTL.

\begin{tabular}{|c|c|c|c|c|}
\hline & Gene set & $\begin{array}{l}\text { Number } \\
\text { of genes }{ }^{\mathrm{a}}\end{array}$ & $P$-value & $\begin{array}{l}\text { Top } 5 \text { genes contributing most } \\
\text { to multivariate expression variability } \\
\text { of the gene set }\end{array}$ \\
\hline 1 & GO_PHOSPHATIDYLINOSITOL_3_KINASE_COMPLEX & $15(20)$ & $7.47 \mathrm{E}-05$ & PIK3R1, PIK3R4, NRBF2, PIK3CA, PIK3C3 \\
\hline $3^{\mathrm{b}}$ & GO_CEREBELLAR_CORTEX_MORPHOGENESIS & $23(30)$ & $1.85 \mathrm{E}-04$ & KNDC1, SPTBN2, LDB1, SERPINE2, FAIM2 \\
\hline 4 & GO_REGULATION_OF_ENDOPLASMIC_RETICULUM_UNFOLDED_PROTEIN_RESPONSE & $22(28)$ & $2.48 \mathrm{E}-04$ & PIK3R1, DAB2IP, HSPA5, BBC3, РОMT2 \\
\hline 5 & GO_GENETIC_IMPRINTING & $15(20)$ & $4.42 \mathrm{E}-04$ & ARID4A, BRCA1, ARID4B, ZFP57, MECP2 \\
\hline 8 & GO_INSULIN_LIKE_GROWTH_FACTOR_RECEPTOR_SIGNALING_PATHWAY & $14(14)$ & $7.87 \mathrm{E}-04$ & PIK3R1, TSC2, GRB10, AKT1, IGF2R \\
\hline 9 & GO_LIPOPROTEIN_PARTICLE_RECEPTOR_ACTIVITY & $12(16)$ & $9.76 \mathrm{E}-04$ & LDLR, LRP6, OLR1, SCARB1, LRP10 \\
\hline 10 & GO_RNA_POLYMERASE_II_ACTIVATING_TRANSCRIPTION_FACTOR_BINDING & $24(36)$ & $9.83 \mathrm{E}-04$ & LDB1, BHLHE40, BEX1, RB1, NCOR1 \\
\hline 11 & GO_FOLIC_ACID_CONTAINING_COMPOUND_METABOLIC_PROCESS & $22(29)$ & $1.10 \mathrm{E}-03$ & MTHFD1, MTRR, ALDH1L1, SLC19A1, FTCD \\
\hline $12^{\mathrm{b}}$ & GO_CEREBELLAR_CORTEX_FORMATION & $19(22)$ & $1.74 \mathrm{E}-03$ & KNDC1, LDB1, CDK5, FAIM2, CEND1 \\
\hline 17 & GO_NEGATIVE_REGULATION_OF_EPITHELIAL_CELL_APOPTOTIC_PROCESS & $18(35)$ & $3.55 \mathrm{E}-03$ & SEMA5A, SCG2, WFS1, TEK, KRIT1 \\
\hline 18 & GO_PYRIMIDINE_DEOXYRIBONUCLEOTIDE_METABOLIC_PROCESS & $14(18)$ & $4.02 \mathrm{E}-03$ & NT5C, MBD4, NEIL2, DTYMK, UNG \\
\hline 19 & GO_NEGATIVE_REGULATION_OF_CIRCADIAN_RHYTHM & $11(17)$ & $4.14 \mathrm{E}-03$ & ADORA1, CRY2, PER2, SUV39H2, SIN3A \\
\hline 20 & GO_NEGATIVE_REGULATION_OF_OXIDOREDUCTASE_ACTIVITY & $13(26)$ & $4.29 \mathrm{E}-03$ & NFKB1, ATP2B4, TMLHE, CNR1, PRDX5 \\
\hline 21 & GO_ACTIVATING_TRANSCRIPTION_FACTOR_BINDING & $40(57)$ & $4.51 \mathrm{E}-03$ & LDB1, BHLHE40, RB1, BEX1, NCOR1 \\
\hline 22 & GO_FOUR_WAY_JUNCTION_DNA_BINDING & $13(15)$ & 4.97E-03 & HMGB3, MECP2, HMGB1, RAD51C, MSH6 \\
\hline $23^{\mathrm{b}}$ & GO_CELL_DIFFERENTIATION_IN_HINDBRAIN & $15(21)$ & $5.24 \mathrm{E}-03$ & KNDC1, LDB1, FAIM2, CEND1, CACNA1A \\
\hline $24^{\mathrm{b}}$ & GO_CEREBELLAR_PURKINJE_CELL_LAYER_MORPHOGENESIS & $11(14)$ & $5.59 \mathrm{E}-03$ & LDB1, SPTBN2, FAIM2, CACNA1A, ATP2B2 \\
\hline 25 & GO_ESTABLISHMENT_OF_MITOTIC_SPINDLE_ORIENTATION & $16(20)$ & $6.20 \mathrm{E}-03$ & HTT, SPRY1, SPRY2, PAFAH1B1, NUMA1 \\
\hline 26 & GO_PTERIDINE_CONTAINING_COMPOUND_METABOLIC_PROCESS & $28(36)$ & $6.64 \mathrm{E}-03$ & PTS, MTR, MTHFD1, MTRR, ALDH1L1 \\
\hline $27^{\mathrm{b}}$ & GO_HINDBRAIN_MORPHOGENESIS & $29(40)$ & $7.09 \mathrm{E}-03$ & KNDC1, SPTBN2, LRP6, FAIM2, LDB1 \\
\hline $28^{\mathrm{b}}$ & GO_REGULATION_OF_ASTROCYTE_DIFFERENTIATION & $19(27)$ & $7.42 \mathrm{E}-03$ & BMP2, PRPF19, SERPINE2, NOTCH1, NF1 \\
\hline
\end{tabular}



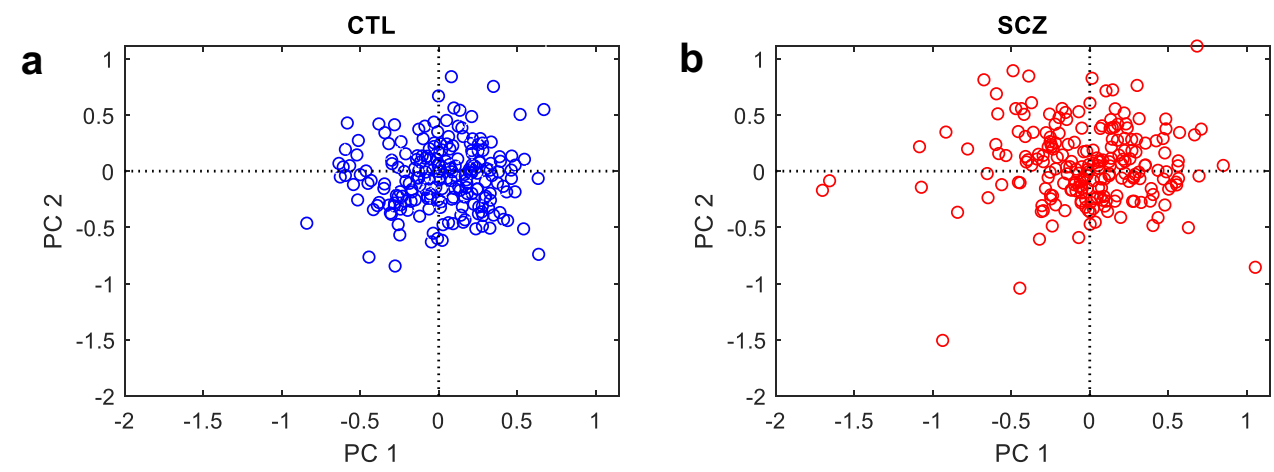

C

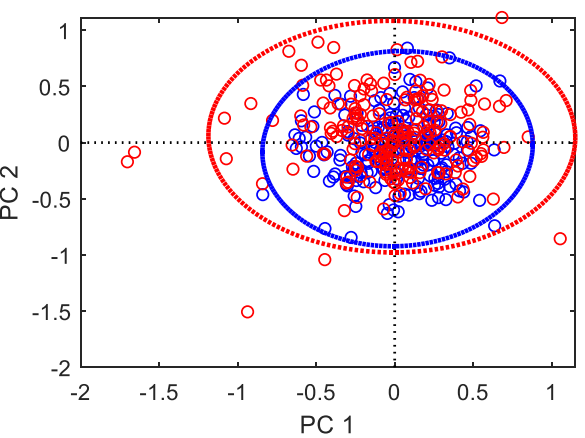

d

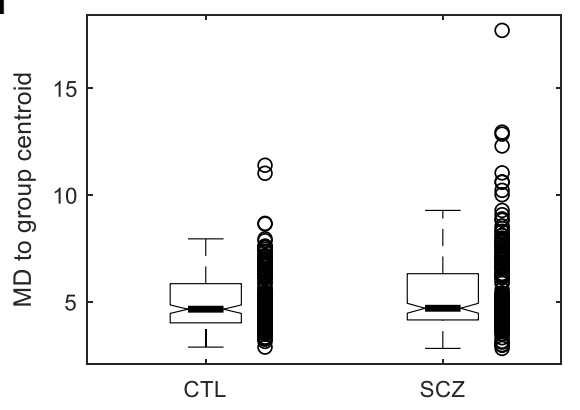

Fig. 2 Gene set, cerebellar cortex morphogenesis, show more dispersed expression in SCZ. The PCA analysis was performed with the gene set expression matrix of pooled samples that contain all SCZ and CTL samples. a Distribution of CTL samples on the PCA space defined by the first two PCs. SCZ samples are made invisible by plotting in white color. b Distribution of SCZ samples with CTL samples made invisible. c Distribution of all samples in the PCA space. Dashed lines indicate the $99 \%$ confidence ellipses. d Boxplot of MD vectors in SCZ and CTL groups, showing the high within-group variance in SCZ.

an example (Fig. 2). We extracted the expression data of 23 genes in the gene set, pooled SCZ with CTL samples, and performed principal component (PC) analysis. On the first and second PC space, SCZ samples are more dispersedly distributed than CTL samples (Fig. 2a-c). Accordingly, the variance in the MD of individual SCZ samples to the centroid is significantly greater than that of CTL samples (Fig. 2d).

We also identified nine gene sets showing the opposite pattern, i.e., smaller expression variance in SCZ than CTL (Supplementary Table 3). These include two related to CNS: (1) neurexin family protein binding and (2) neuron cell-cell adhesion.

\section{Statistical power analysis}

We used computer simulations to conduct a power analysis for our MD-based multivariate test for homogeneity of variances. As mentioned, our test is an extension of the procedure of Anderson $^{48}$. The simulations were done with combinations of a series of sample sizes and varying levels of variance difference between case and control groups (see Methods for details). We considered a balanced design with the sample size of the case group equals to that of the control group. The result of the power analysis shows that the power of our extended test starts to increase when the sample size per group is over 800 (Supplementary Fig. 5a). The test becomes highly sensitive when the sample size per group reaches 1000 -in this case, when variances of the two groups differ by twofold, the statistical power of our test can reach $80 \%$. To increase the power of our MD-based test, we proposed a modified version of it (see Methods). With this modification, the power of our test increases substantially (Supplementary Fig. 5b). Our modified MD-based test requires $\sim 700$ samples to reach $80 \%$ power. Despite this improvement, the power analysis suggests that the sample size we used in our real data analysis (212 and 214 for SCZ and CTL, respectively) was still too small. Additional studies are needed to investigate differences in gene set expression variability between groups further.

Genetic variants contribute to dispersed gene expression in SCZ To assess the contribution of genetic variants to the gene expression dispersion in SCZ, we conducted the expression variability QTL (evQTL) mapping analysis ${ }^{63,64}$. An evQTL is an SNP with alleles associated with group variance in gene expression. In this case-control study setting, we were more interested in SNPs with different effects on SCZ and CTL, and thus we set out to identify SCZ-specific evQTLs. We tested all common SNPs segregating in SCZ and $C T L$, i.e., minor allele frequency (MAF) $>0.15$ in both populations, to identify those with genotypes associated with gene expression variance in SCZ $(p<1 \mathrm{e}-7, \mathrm{~B}-\mathrm{F}$ test) but not in CTL ( $p>0.05, \mathrm{~B}-\mathrm{F}$ test, Fig. 3a). We identified 2503 SCZ-specific evQTLs involving 1453 distinct autosomal proteincoding genes (Supplementary Table 4, see Supplementary Fig. 6 for more examples). For comparison, we used the same procedure and $p$-value cutoffs to identify CTL-specific evQTLs with SCZ samples as the background group. We identified 2076 CTL-specific evQTLs involving 1277 genes. Although the number of CTLspecific evQTLs is comparable to that of SCZ-specific evQTLs, a $\mathrm{q}-\mathrm{q}$ plot shows that the overall statistical significance is much stronger for SCZ-specific evQTLs, especially for those highly significant ones (Fig. 3b). We plotted the links between these highly significant evQTLs $(p<1 \mathrm{e}-9)$ with their target genes for both SCZ and CTL results. Most of these relationships are transacting and more SCZ-specific evQTLs connect with target genes and form a denser picture than CTL-specific ones do (Fig. 3c).

\section{DISCUSSION}

Our analysis focuses on the differences in expression variances between groups. The DV approach we adopted here could be 
a

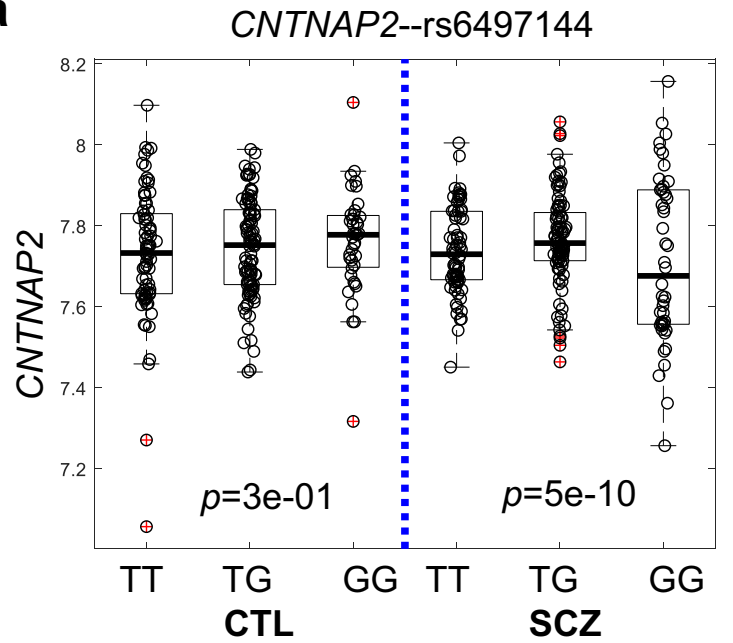

COCH--rs713989

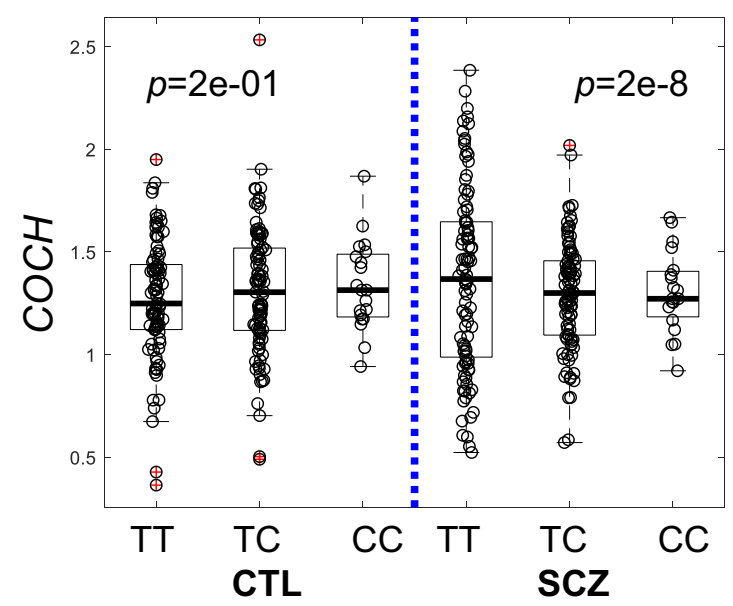

b

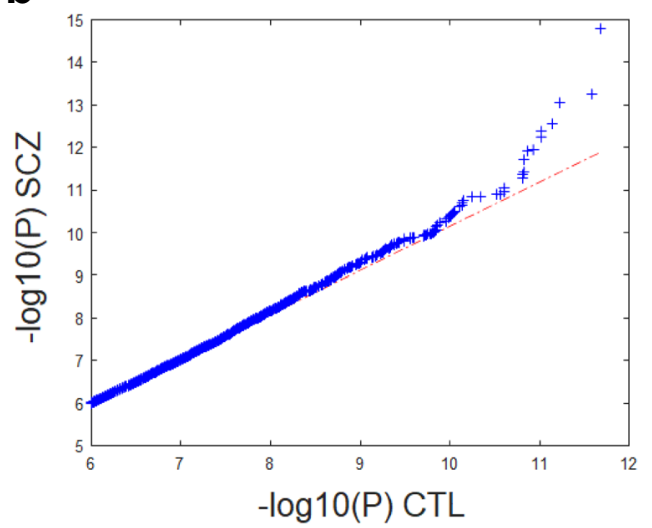

C

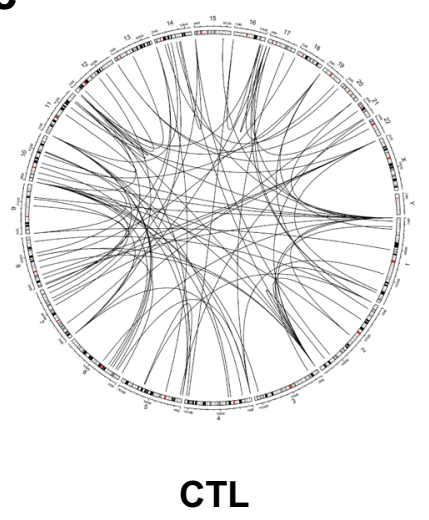

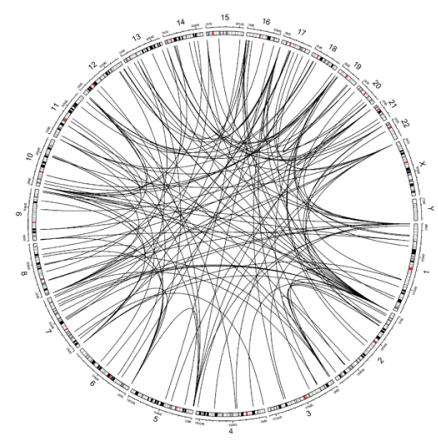

SCZ

Fig. 3 Genetic variants associated with gene expression variability in SCZ. a Two examples of SCZ-specific evQTLs, showing significant differences in expression variances between genotypes in SCZ but not in CTL. P-values of the B-F test for three genotype groups in SCZ and CTL, respectively, are given. $\mathbf{b}$ Quantile-quantile plot of $P$-values for evQTL associations in SCZ ( $y$-axis) against those in CTL ( $x$-axis). $\mathbf{c}$ Difference in the density of highly significant evQTL associations between variants and genes in SCZ and CTL.

rooted in the theory of dynamics of correlation and variance in systems under the load of environmental factors ${ }^{65}$. For heterogonous disorders like SCZ, we show that the DV approach is powerful in identifying significant genes and characterizing biological pathways and processes critical to the disorder. DV approach is complementary to the more commonly used DE method. So far, identified DE genes between SCZ and CTL show tremendous functional diversity and often fail to form supported, functionally interpretable gene sets or pathways (with few exceptions such as the AMPA GluR1 receptor subunits and the glutamatergic neurotransmission pathway) ${ }^{66}$, which is not unexpected under the assumption of substantial heterogeneity in SCZ pathophysiology ${ }^{32}$.

Overdispersion is the term we borrow from statistics to describe the presence of greater variability (statistical dispersion) in gene expression data in SCZ than would be expected based on that in CTL. The overdispersion pattern we observed in SCZ is in line with the fact that clinical heterogeneity amongst people diagnosed with SCZ is high, which has hampered the development of individual treatment and research into new treatment strategies. We assume that key pathways underlying SCZ risk are disrupted via many different causes-genetic, epigenetic, and environmental. The consequence of these disruptions is collectively reflected as dysregulated gene expression, which is, in turn, characterized by an increased level of group dispersions (variances). Thus, the degree of gene expression dispersion is an excellent predictor of functional disruptions. Even if the illness of SCZ in every affected individual arises from a different specific cause, each will nonetheless share disruption of related key biological processes.

Furthermore, the effects of environmental factors may accumulate throughout the progress of chronic SCZ. In such cases, a lifetime of stress, smoking, and antipsychotic drug administration are examples of these environmental factors, which may take effect through interacting with genotypes of individual SCZ patients. Thus, through assessing the gene expression in the brain tissue, we identify the related pathways dysregulated in patients affected by SCZ. Such dysregulated expression is a transcriptional echo of the pathways and mechanisms associated with the onset of the illness.

In this study, VEGFA, the gene encoding vascular endothelial growth factor, stands out as the most significant single DV gene, cross-validated with two independent expression data sets ${ }^{29,42}$. The discovery of this significant gene is consistent with accumulating evidence indicating that SCZ is accompanied by abnormal vascularization ${ }^{38,67,68}$. The role of VEGF in causing neurovascular dysfunction has been known to be correlated with hypoxia-ischemia insults during early life and is strongly associated with cognitive dysfunction ${ }^{38,41}$. Clinical studies examining peripheral VEGF levels in SCZ versus CTL have yielded conflicting results. While some studies found elevated serum VEGF 
concentrations in individuals with $\mathrm{SCZ}^{69,70}$, others revealed no significant difference $\mathrm{e}^{71-74}$, or lower concentrations ${ }^{75,76}$. The extremely high between-individual variability in VEGFA expression may explain such conflicting findings.

One of the caveats of our study is that the influence of antipsychotics on gene expression in SCZ patients could not be easily controlled. Antipsychotic drugs are widely used to treat SCZ. In the clinical settings, because antipsychotics have large interindividual differences in efficacy, there is no methodology to predict the effect of antipsychotics ${ }^{77}$. Although much progress has been made on antipsychotic drug development, precise mechanisms behind the action of antipsychotics are poorly understood $^{78,79}$. Non-genetic factors such as epigenetic modifications are known to be involved in the effect of antipsychotics ${ }^{80}$. For VEGFA, it is known that antipsychotics influences plasma VEGF levels, but the direction of the influence varies (refs ${ }^{76,81}$. cf. ref. ${ }^{72}$ ).

An important contribution of our study is to develop the homogeneity test of multivariate dispersions further using an MDbased extension. The initially proposed procedure by Anderson (ref. ${ }^{48}$ ) is flexible enough to allow any distance measure to be adopted. We have previously used a slightly different implementation of the test to identify dysregulated gene sets in autism spectrum disorder ${ }^{82,83}$. Other implementations include the one based on the means of within-group distances, which does not require group center calculations to obtain the distance statistic ${ }^{84}$, and the one to compare $k$ populations based on Fréchet variance for general metric space valued data objects, with emphasis on comparing means and variances ${ }^{85}$. The most important feature shared by these tests is their capability of capturing the betweengroup difference in multivariate covariance of variables. Our method, when applied to gene sets, has proved to be useful in identifying functionally meaningful gene sets. Our results reveal that transcriptional dysregulation in genes responsible for brain development is significantly implicated in SCZ, which is in line with the consensus of SCZ being a brain disorder ${ }^{11,16,55}$. Our analysis with gene sets supports that the cell-death pathways activated via extrasynaptic NMDA receptors ${ }^{86,87}$, folic acid ${ }^{88,89}$, and metallopeptidases ${ }^{90}$, are implicated in SCZ.

We also report the opposite pattern detected in several gene sets, for which expression variability is reduced rather than elevated in SCZ. These include genes with the molecular function of interacting selectively and non-covalently with neurexins that act as cell recognition molecules at nerve terminals. Thus, despite its scarcity, such an underdispersion pattern in gene expression variability may also have important implications compared with the overdispersion pattern. The underdispersion pattern may even reflect a more severely dysregulated status of gene expression in SCZ.

We explored the relationship between genetic variants and expression variability. Previously, we adopted the ' $\mathrm{VQTL}$ ' model ${ }^{91}$, and applied the model-based method to human gene expression data ${ }^{63}$. Up to date, a large number of evQTLs have been identified with different human gene expression data sets ${ }^{64,92,93}$. Here, in the present study, we detect evQTLs in SCZ and CTL samples, separately, and highlight differences in the number and statistical significance of evQTLs between SCZ and CTL. We found more statistically significant evQTLs in SCZ than in CTL, suggesting that common genetic variants may play a more important role in shaping the gene expression variability in SCZ. It is not clear though whether these genetic variants exert destabilizing function on their own or act through interacting with each other ${ }^{92}$. Nevertheless, these results have clinical implications. For example, CALM1-rs2123259 (GT) and HTR1A-rs12440923 (TC) are two SCZspecific evQTLs identified in this study (Supplementary Table 3). CALM1 encodes for calmodulin 1 and HTR1A, serotonin $1 \mathrm{~A}$ receptor (or $5-\mathrm{HT}_{1 \mathrm{~A}}$ receptor). Both gene products are targets of antipsychotic drugs. For example, aripiprazole is a partial agonist at the $5-\mathrm{HT}_{1 \mathrm{~A}}$ receptor; chlorpromazine binds to calmodulin to exert an inhibitory effect and exhibited antagonist activity at serotonin $1 \mathrm{~A}$ receptors ${ }^{94,95}$. According to the evQTL pattern, the expression level of CALM1 in SCZ patients with TT genotype at rs2123259 (chr15:87257140_hg19) is highly variable from patient to patient, and the same as for the HTR1A expression in patients with CC genotype at rs12440923 (chr15:93164993_hg19). This information ought to be taken into account in antipsychotic medication administration.

In summary, we proposed new analytical methods to reveal overdispersed gene expression in SCZ. These methods are based on statistical tests for homogeneity in group variances. We showed the application of our methods in the discovery of genetic underpinnings of SCZ that are notoriously difficult to determine. We identified single genes as well as gene sets showing greater gene expression variability in SCZ patients but not in unaffected controls. Functional interpretation of these genes points us to the dysregulation of brain function pertaining to a number of new mechanisms. The highly variable expression of these genes in $\mathrm{SCZ}$ is associated with the heterogeneous nature of this disorder, which is characterized by varying clinic presentations and individualized symptoms. As technologies for high-throughput data generation become more common and affordable, the sample size per study will continually increase, which will greatly improve the power of variance-based statistical inference. Given these, we anticipate our study inspires new conceptual development toward variability-centric analyses in SCZ research.

\section{METHODS}

\section{Ethical statement}

This study was exempt from the institutional review board (IRB) review at Texas A\&M University; no identifiable private information or identifiable biospecimen was used in the analyses.

\section{Data sets}

The gene-level expression data used for the analyses in this study was generated as part of the CommonMind Consortium (CMC) study ${ }^{29}$. The expression matrix was derived from the raw read count matrix through a series of normalization and adjustment. Briefly, 16,423 genes with at least one CPM (read counts per million total reads) in at least $50 \%$ of the individuals were retained and processed. The initial normalization was done using voom ${ }^{96}$. Weighted linear regression was then performed for each gene to control for known covariates. The data was further adjusted for hidden variables detected by surrogate variable analysis (see ref. ${ }^{29}$ for details). We downloaded the processed expression data matrix from the CMC Knowledge Portal (see Data availability). From this data matrix, we extracted data generated from European ancestry individuals, including 212 SCZ and 214 CTL samples. The SCZ group contains 77 female (35.8\%) and 137 male (64.2\%) donors; the CTL group that contains 91 female (42.9\%) and 121 male (57.1\%) donors (see Supplementary Table 5 for more details). We used this European data set throughout all subsequent analyses. In addition to the CMC data, an independent, microarray-based gene expression data, collected by a study of multiple psychiatric disorders $^{42}$, was obtained. From this microarray data set, we extracted expression data for 139 SCZ and 266 CTL samples. We also obtained the data from the Phase1 study (http://eqtl.brainseq.org/phase1) of the Brainseq project ${ }^{36}$. This data set contains information about the use of antipsychotic medications, allowing the investigation of the influence of medications on gene expression variability among SCZ patients.

Test for homogeneity of expression variances of single genes Brown-Forsythe (B-F) test was applied to each gene to test for the significant difference in expression variance between subjects of the SCZ and CTL groups. B-F test is a statistical analysis related to Levene's testboth are tests for homogeneity of variance. B-F test involves determining an absolute deviation score from group medians, while Levene's test from the group means. After running the B-F test for all individual genes, we used the Benjamini-Hochberg $(\mathrm{B}-\mathrm{H})$ procedure to control the FDR of hypothesis tests ${ }^{97}$. 
MD-based multivariate test for homogeneity of expression variances of gene sets

Anderson (2006) proposed a distance-based test of homogeneity of multivariate dispersions for a one-way ANOVA design ${ }^{48}$. To identify gene sets with differential multivariate expression variability between SCZ and CTL, we adopted the 'Anderson06' test and applied it to functional gene sets. These functional gene sets are pre-defined in the MSigDB v5.2 ${ }^{98}$. We selected those defined with $\mathrm{GO}$ ontologies of biological process and molecular function. For a given gene set, we measured multivariate expression dispersion (variance) of genes in SCZ and CTL separately. We calculated Mahalanobis distance (MD) of each SCZ and CTL individuals to their group centroids in the multivariate space. Compared with the Euclidean distance used in the original Anderson06 test, MD is a more appropriate distance metric for gene set expression, because expression levels of different genes in a gene set are likely to be correlated ${ }^{49,50}$. To test if the dispersions (variances) of SCZ and CTL groups are different, MDs of group members to the group centroid were subject to ANOVA ${ }^{48}$. We call our method the MD-based Anderson06 test. To increase the statistical power, we introduced a modified version of the MD-based Anderson06 test. In the modified version, values of MD distance metric obtained from all samples (SCZ and CTL) were pooled, and the top $90 \%$ percentile of values were multiplicated with a constant that equals to the maximum of $M D$ values, keeping the rest of MD values unchanged. After this treatment, the rest of the steps such as the ANOVA test remained the same as described above. This modification was found to be able to increase the power of the test markedly (Supplementary Fig. 4). Therefore, the modified version was used throughout our analyses for gene sets. Finally, to determine which genes in a gene set have the most influence on the MD, we used the method proposed by Garthwaite and Koch ${ }^{62}$.

\section{Power analyses}

We conducted simulations to evaluate the power of the MD-based Anderson06 test. We fixed the size of the input gene set at 23, based on the number of genes in the actual gene set of GO_CEREBELLAR_CORTEX_MORPHOGENESIS (Table 1). We also fixed the covariance matrices, $\Sigma_{0}$ and $\bar{\Sigma}_{1}$ of the input gene set for CTL and SCZ samples, respectively, using the values calculated from the actual expression data of the gene set. We varied values of two parameters: (1) the sample size per group, and (2) $a$, a factor multiplied to $\Sigma_{1}$ to increase multivariate variability and assessed the power as a function of these two parameters. We set the sample size of the SCZ group equals to that of the CTL group. We assume that the gene expression levels of the gene set obey a multivariate normal distribution (MVN). The simulations were done as follows:

Step 1: We calculated the mean vector $\mu_{0}$ and covariance matrix $\Sigma_{0}$ from the expression values of the real gene set in CTL, and $\mu_{1}$ and $\Sigma_{1}$ in SCZ.

Step 2: We generated the simulated expression data of CTL samples from $\operatorname{MVN}\left(\mu_{0}, \Sigma_{0}\right)$. We generated the expression data of SCZ samples from $\operatorname{MVN}\left(\mu_{1},\left(1+e^{\prime}\right) \Sigma_{1}\right)$, where / obeys uniform distribution $(0, a)$.

Step 3: We set $a=1,1.2, \ldots, 5$ and the sample size $n=100,200, \ldots$, 2000. For each pair of the two parameters, we simulated expression data matrix $X_{1}$ for SCZ. Accordingly, we simulated the expression matrix $X_{0}$ for CTL. For samples in the data $X_{1}$, we calculated their MD to the group centroid and obtained the vector $D_{1}$ for SCZ. In the same way, we obtained the vector $D_{0}$ for CTL. We used ANOVA to compare the difference between $D_{1}$ and $D_{0}$. For each pair of $a$ and $n$, this process was repeated 100 times and the number of ANOVA $p<0.01$ was recorded as the probability that the test correctly rejects the null hypothesis. We used the same procedure described above to perform the power analysis for the modified version of the MD-based Anderson06 test.

\section{Identification of SCZ-specific evQTLs}

The genotype data of the SCZ and CTL samples was obtained from the CMC Knowledge Portal web site (see Data availability). For each SNP, the MAF is calculated for SCZ and CTL samples separately. The SNPs with both $\mathrm{MAF}>0.15$ were retained for the analysis. B-F tests were conducted with three genotype groups of each SNP to examine whether there is a significant difference in expression variances between any two groups. The B-F test was conducted for SCZ and CTL samples separately. The SCZspecific evQTLs were called when the $p<1 \mathrm{e}-7$ in SCZ samples and $p>$ 0.05 in CTL samples; the CTL-specific evQTLs were called when the $p<1 \mathrm{e}-7$ in CTL samples and $p>0.05$ in SCZ samples. SZGR 2.0 (ref. ${ }^{99}$ ) was used to identify antipsychotic drugs whose targets are evQTL genes.
Reporting summary

Further information on research design is available in the Nature Research Reporting Summary linked to this article.

\section{DATA AVAILABILITY}

The main data set used for the analyses in this study was generated as part of the CMC supported by funding from Takeda Pharmaceuticals Company Limited, F Hoffman-La Roche and NIH Grants R01MH085542, R01MH093725, P50MH066392, P50MH080405, R01MH097276, RO1-MH-075916, P50M096891, P50MH084053S1, R37MH057881 and R37MH057881S1, HHSN271201300031C, AG02219, AG05138, and $\mathrm{MH06692}$. The processed expression data matrix can be obtained from the CMC Knowledge Portal web page at https://www.synapse.org/\#!Synapse:syn5609491. The file name is 'CMC_MSSM-Penn-Pitt_DLPFC_mRNA_IlluminaHiSeq2500_gene-adjustedSVA-dataNormalization-noAncestry-adjustedLogCPM', which is the one used for eQTL identification in the original CMC study ${ }^{29}$. The genotype data of SCZ and CTL samples is available at https://www.synapse.org//\#!Synapse:syn3275211.

\section{CODE AVAILABILITY}

Source code to deterministically generate all results and plots in this paper can be found at https://github.com/cailab-tamu/scz_ge_dispersion.

Received: 31 August 2019; Accepted: 13 February 2020; Published online: 03 April 2020

\section{REFERENCES}

1. Saha, S., Chant, D., Welham, J. \& McGrath, J. A systematic review of the prevalence of schizophrenia. PLoS Med. 2, e141 (2005).

2. McGrath, J., Saha, S., Chant, D. \& Welham, J. Schizophrenia: a concise overview of incidence, prevalence, and mortality. Epidemiol. Rev. 30, 67-76 (2008).

3. Knapp, M., Mangalore, R. \& Simon, J. The global costs of schizophrenia. Schizophr. Bull. 30, 279-293 (2004).

4. Picardi, A. et al. Heterogeneity and symptom structure of schizophrenia. Psychiatry Res. 198, 386-394 (2012).

5. Andreasen, N. C., Flaum, M., Schultz, S., Duzyurek, S. \& Miller, D. Diagnosis, methodology and subtypes of schizophrenia. Neuropsychobiology 35, 61-63 (1997).

6. Kay, S. R. \& Sevy, S. Pyramidical model of schizophrenia. Schizophr. Bull. 16, 537-545 (1990).

7. Budde, M. et al. A longitudinal approach to biological psychiatric research: The PsyCourse study. Am. J. Med. Genet. B, https://doi.org/10.1002/ajmg.b.32639 (2018).

8. McGrath, J. Dissecting the heterogeneity of schizophrenia outcomes. Schizophr. Bull. 34, 247-248 (2008).

9. Andreasen, N. C. et al. Remission in schizophrenia: proposed criteria and rationale for consensus. Am. J. Psychiatry 162, 441-449 (2005).

10. Murray, R. M., O'Callaghan, E., Castle, D. J. \& Lewis, S. W. A neurodevelopmental approach to the classification of schizophrenia. Schizophr. Bull. 18, 319-332 (1992).

11. Demjaha, A., MacCabe, J. H. \& Murray, R. M. How genes and environmental factors determine the different neurodevelopmental trajectories of schizophrenia and bipolar disorder. Schizophr. Bull. 38, 209-214 (2012).

12. Kaymaz, N. \& van Os, J. Murray et al. (2004) revisited: is bipolar disorder identical to schizophrenia without developmental impairment? Acta Psychiatr. Scand. 120, 249-252 (2009)

13. Arnedo, J. et al. Decomposition of brain diffusion imaging data uncovers latent schizophrenias with distinct patterns of white matter anisotropy. Neuroimage 120, 43-54 (2015).

14. Cernis, E. et al. Schizophrenia patients with high intelligence: a clinically distinct sub-type of schizophrenia? Eur. Psychiatry 30, 628-632 (2015).

15. MacCabe, J. H. et al. Superior intellectual ability in schizophrenia: neuropsychological characteristics. Neuropsychology 26, 181-190 (2012).

16. Karlsgodt, K. H., Sun, D. \& Cannon, T. D. Structural and functional brain abnormalities in schizophrenia. Curr. Dir. Psychol. Sci. 19, 226-231 (2010).

17. van Os, J. "Schizophrenia" does not exist. BMJ 352, i375 (2016).

18. Lasalvia, A., Penta, E., Sartorius, N. \& Henderson, S. Should the label "schizophrenia" be abandoned? Schizophr. Res. 162, 276-284 (2015).

19. Sommer, I. E. \& Carpenter, W. T. Schizophrenia: changing the name and broadening the concept is problematic. BMJ 352, i1080 (2016)

20. Hilker, R. et al. Heritability of schizophrenia and schizophrenia spectrum based on the nationwide Danish twin register. Biol. Psychiatry 83, 492-498 (2018). 
21. Schizophrenia Psychiatric Genome-Wide Association Study, C. Genome-wide association study identifies five new schizophrenia loci. Nat. Genet. 43, 969-976 (2011).

22. Schizophrenia Working Group of the Psychiatric Genomics, C. Biological insights from 108 schizophrenia-associated genetic loci. Nature 511, 421-427 (2014).

23. Ripke, S. et al. Genome-wide association analysis identifies 13 new risk loci for schizophrenia. Nat. Genet. 45, 1150-1159 (2013).

24. Walsh, T. et al. Rare structural variants disrupt multiple genes in neurodevelopmental pathways in schizophrenia. Science 320, 539-543 (2008).

25. Xu, B. et al. Strong association of de novo copy number mutations with sporadic schizophrenia. Nat. Genet 40, 880-885 (2008).

26. Stefansson, H. et al. Large recurrent microdeletions associated with schizophrenia. Nature 455, 232-236 (2008).

27. International Schizophrenia, C. Rare chromosomal deletions and duplications increase risk of schizophrenia. Nature 455, 237-241 (2008).

28. Sullivan, P. F., Daly, M. J. \& O'Donovan, M. Genetic architectures of psychiatric disorders: the emerging picture and its implications. Nat. Rev. Genet. 13, 537-551 (2012).

29. Fromer, M. et al. Gene expression elucidates functional impact of polygenic risk for schizophrenia. Nat. Neurosci. 19, 1442-1453 (2016).

30. Gusev, A. et al. Transcriptome-wide association study of schizophrenia and chromatin activity yields mechanistic disease insights. Nat. Genet. 50, 538-548 (2018).

31. Pardinas, A. F. et al. Common schizophrenia alleles are enriched in mutationintolerant genes and in regions under strong background selection. Nat. Genet. 50, 381-389 (2018).

32. Sanders, A. R. et al. Transcriptome study of differential expression in schizophrenia. Hum. Mol. Genet. 22, 5001-5014 (2013).

33. Fillman, S. G. et al. Increased inflammatory markers identified in the dorsolateral prefrontal cortex of individuals with schizophrenia. Mol. Psychiatry 18, 206-214 (2013).

34. Andreasen, N. C. A unitary model of schizophrenia: Bleuler's "fragmented phrene" as schizencephaly. Arch. Gen. Psychiatry 56, 781-787 (1999).

35. Brown, M. B. \& Forsythe, A. B. Robust tests for the equality of variances. J. Am. Stat. Assoc. 69, 364-367 (1974).

36. Jaffe, A. E. et al. Developmental and genetic regulation of the human cortex transcriptome illuminate schizophrenia pathogenesis. Nat. Neurosci. 21, 1117-1125 (2018).

37. Huang da, W., Sherman, B. T. \& Lempicki, R. A. Systematic and integrative analysis of large gene lists using DAVID bioinformatics resources. Nat. Protoc. 4, 44-57 (2009).

38. Misiak, B., Stramecki, F., Stanczykiewicz, B., Frydecka, D. \& Lubeiro, A. Vascular endothelial growth factor in patients with schizophrenia: A systematic review and meta-analysis. Prog. Neuropsychopharmacol. Biol. Psychiatry 86, 24-29 (2018).

39. Sun, Y. et al. VEGF-induced neuroprotection, neurogenesis, and angiogenesis after focal cerebral ischemia. J. Clin. Invest. 111, 1843-1851 (2003).

40. Jin, K. et al. Vascular endothelial growth factor (VEGF) stimulates neurogenesis in vitro and in vivo. Proc. Natl Acad. Sci. USA 99, 11946-11950 (2002).

41. Howell, K. R. \& Armstrong, J. Vascular endothelial growth factor (VEGF) in neurodevelopmental disorders. Curr. Behav. Neurosci. Rep. 4, 299-308 (2017).

42. Gandal, M. J. et al. Shared molecular neuropathology across major psychiatric disorders parallels polygenic overlap. Science 359, 693-697 (2018).

43. Wang, K. S., Liu, X. F. \& Aragam, N. A genome-wide meta-analysis identifies novel loci associated with schizophrenia and bipolar disorder. Schizophr. Res. 124, 192-199 (2010).

44. Shi, Y. et al. Common variants on $8 \mathrm{p} 12$ and $1 \mathrm{q} 24.2$ confer risk of schizophrenia. Nat. Genet. 43, 1224-1227 (2011).

45. Goes, F. S. et al. Genome-wide association study of schizophrenia in Ashkenazi Jews. Am. J. Med. Genet. Part B, Neuropsychiatr. Genet. 168, 649-659 (2015).

46. Lencer, R. et al. Genome-wide association studies of smooth pursuit and antisaccade eye movements in psychotic disorders: findings from the B-SNIP study. Transl. Psychiatry 7, e1249 (2017).

47. Autism Spectrum Disorders Working Group of The Psychiatric Genomics, C. Metaanalysis of GWAS of over 16,000 individuals with autism spectrum disorder highlights a novel locus at 10q24.32 and a significant overlap with schizophrenia. Mol. Autism 8, 21 (2017).

48. Anderson, M. J. Distance-based tests for homogeneity of multivariate dispersions. Biometrics 62, 245-253 (2006).

49. Zeng, Y. et al. Aberrant gene expression in humans. PLoS Genet. 11, https://doi. org/10.1371/journal.pgen.1004942 (2015).

50. Brinkmeyer-Langford, C. L., Guan, J., Ji, G. \& Cai, J. J. Aging shapes the populationmean and -dispersion of gene expression in human brains. Front Aging Neurosci. 8, 183 (2016).

51. Enriquez-Barreto, L. \& Morales, M. The PI3K signaling pathway as a pharmacological target in Autism related disorders and Schizophrenia. Mol. Cell Ther. 4, 2 (2016).
52. Kordi-Tamandani, D. M. \& Mir, A. Relationship between phosphoinositide-3kinase genetic polymorphism and schizophrenia. Nord J. Psychiatry 70, 272-275 (2016).

53. Carrard, A. et al. Genetic association of the Phosphoinositide-3 kinase in schizophrenia and bipolar disorder and interaction with a BDNF gene polymorphism. Brain Behav. 1, 119-124 (2011).

54. Law, A. J. et al. Neuregulin 1-ErbB4-PI3K signaling in schizophrenia and phosphoinositide 3-kinase-p110delta inhibition as a potential therapeutic strategy. Proc. Natl Acad. Sci. USA 109, 12165-12170 (2012).

55. Kelly, S. et al. Widespread white matter microstructural differences in schizophrenia across 4322 individuals: results from the ENIGMA Schizophrenia DTI Working Group. Mol. Psychiatry 23, 1261-1269 (2018).

56. Jarskog, L. F., Glantz, L. A., Gilmore, J. H. \& Lieberman, J. A. Apoptotic mechanisms in the pathophysiology of schizophrenia. Prog. Neuropsychopharmacol. Biol. Psychiatry 29, 846-858 (2005).

57. Catts, V. S. \& Weickert, C. S. Gene expression analysis implicates a death receptor pathway in schizophrenia pathology. PLOS ONE 7, e35511 (2012).

58. Wang, S. \& Kaufman, R. J. The impact of the unfolded protein response on human disease. J. Cell Biol. 197, 857-867 (2012).

59. Saedisomeolia, A., Djalali, M., Moghadam, A. M., Ramezankhani, O. \& Najmi, L. Folate and vitamin B12 status in schizophrenic patients. J. Res. Med. Sci. 16, S437-S441 (2011).

60. Brown, H. E. \& Roffman, J. L. Vitamin supplementation in the treatment of schizophrenia. CNS Drugs 28, 611-622 (2014).

61. Roffman, J. L. et al. Randomized multicenter investigation of folate plus vitamin B12 supplementation in schizophrenia. JAMA Psychiatry 70, 481-489 (2013).

62. Garthwaite, P. H. \& Koch, I. Evaluating the contributions of individual variables to a quadratic form. Aust. N. Z. J. Stat. 58, 99-119 (2016).

63. Hulse, A. M. \& Cai, J. J. Genetic variants contribute to gene expression variability in humans. Genetics 193, 95-108 (2013).

64. Wang, G., Yang, E., Brinkmeyer-Langford, C. L. \& Cai, J. J. Additive, epistatic, and environmental effects through the lens of expression variability QTL in a twin cohort. Genetics 196, 413-425 (2014).

65. Gorban, A. N., Smirnova, E. V. \& Tyukina, T. A. Correlations, risk and crisis: from physiology to finance. arXiv https://arxiv.org/abs/0905.0129 (2009).

66. Wiedholz, L. M. et al. Mice lacking the AMPA GluR1 receptor exhibit striatal hyperdopaminergia and 'schizophrenia-related' behaviors. Mol. Psychiatry 13, 631-640 (2008).

67. Hanson, D. R. \& Gottesman, I. I. Theories of schizophrenia: a geneticinflammatory-vascular synthesis. BMC Med. Genet. 6, 7 (2005).

68. Moises, H. W., Wollschlager, D. \& Binder, H. Functional genomics indicate that schizophrenia may be an adult vascular-ischemic disorder. Transl. Psychiatry 5, e616 (2015).

69. Pillai, A. et al. Association of serum VEGF levels with prefrontal cortex volume in schizophrenia. Mol. Psychiatry 21, 686-692 (2016).

70. Balotsev, R. et al. Inflammatory, cardio-metabolic and diabetic profiling of chronic schizophrenia. Eur. Psychiatry 39, 1-10 (2017).

71. Di Nicola, M. et al. Serum and gene expression profile of cytokines in first-episode psychosis. Brain Behav. Immun. 31, 90-95 (2013).

72. Haring, L. et al. Antipsychotic treatment reduces psychotic symptoms and markers of low-grade inflammation in first episode psychosis patients, but increases their body mass index. Schizophr. Res. 169, 22-29 (2015).

73. Lizano, P. L. et al. Angiogenic and immune signatures in plasma of young relatives at familial high-risk for psychosis and first-episode patients: a preliminary study. Schizophr. Res. 170, 115-122 (2016).

74. Murphy, B. P. et al. Vascular endothelial growth factor and brain-derived neurotrophic factor in quetiapine treated first-episode psychosis. Schizophr. Res Treat. 2014, 719395 (2014).

75. Xiao, W. et al. Baseline serum vascular endothelial growth factor levels predict treatment response to antipsychotic medication in patients with schizophrenia. Eur. Neuropsychopharmacol. 28, 603-609 (2018).

76. Lee, B. H. et al. Alterations in plasma vascular endothelial growth factor levels in patients with schizophrenia before and after treatment. Psychiatry Res. 228, 95-99 (2015).

77. Kantor, E. D., Rehm, C. D., Haas, J. S., Chan, A. T. \& Giovannucci, E. L. Trends in prescription drug use among adults in the United States From 1999-2012. JAMA 314, 1818-1831 (2015).

78. Choi, K. H. et al. Effects of typical and atypical antipsychotic drugs on gene expression profiles in the liver of schizophrenia subjects. BMC Psychiatry 9, 57 (2009).

79. Sainz, J., Prieto, C., Ruso-Julve, F. \& Crespo-Facorro, B. Blood gene expression profile predicts response to antipsychotics. Front. Mol. Neurosci. 11, 73 (2018).

80. Seo, M. K. et al. Effects of antipsychotic drugs on the epigenetic modification of brain-derived neurotrophic factor gene expression in the hippocampi of chronic restraint stress rats. Neural Plast. 2018, 2682037 (2018). 
81. Pillai, A. \& Mahadik, S. P. Differential effects of haloperidol and olanzapine on levels of vascular endothelial growth factor and angiogenesis in rat hippocampus. Schizophr. Res. 87, 48-59 (2006).

82. Guan, J. et al. Exploiting aberrant mRNA expression in autism for gene discovery and diagnosis. Hum. Genet. 135, 797-811 (2016).

83. Guan, J., Chen, M., Ye, C., Cai, J. J. \& Ji, G. AEGS: identifying aberrantly expressed gene sets for differential variability analysis. Bioinformatics 34, 881-883 (2018).

84. Gijbels, I. \& Omelka, M. Testing for homogeneity of multivariate dispersions using dissimilarity measures. Biometrics 69, 137-145 (2013).

85. Dubey, P. \& Müller, H.-G. Fréchet analysis of variance for random objects. arXiv https://arxiv.org/abs/1710.02761 (2017).

86. Zhou, Q. \& Sheng, M. NMDA receptors in nervous system diseases. Neuropharmacology 74, 69-75 (2013).

87. Sattler, R. \& Tymianski, M. Molecular mechanisms of glutamate receptormediated excitotoxic neuronal cell death. Mol. Neurobiol. 24, 107-129 (2001).

88. Young, S. N. \& Ghadirian, A. M. Folic acid and psychopathology. Prog. Neuropsychopharmacol. Biol. Psychiatry 13, 841-863 (1989).

89. Kale, A. et al. Reduced folic acid, vitamin B12 and docosahexaenoic acid and increased homocysteine and cortisol in never-medicated schizophrenia patients: implications for altered one-carbon metabolism. Psychiatry Res. 175, 47-53 (2010).

90. Kuzman, M. R., Medved, V., Terzic, J. \& Krainc, D. Genome-wide expression analysis of peripheral blood identifies candidate biomarkers for schizophrenia. J. Psychiatr. Res. 43, 1073-1077 (2009).

91. Ronnegard, L. \& Valdar, W. Detecting major genetic loci controlling phenotypic variability in experimental crosses. Genetics 188, 435-447 (2011).

92. Yang, E. et al. Epistasis and destabilizing mutations shape gene expression variability in humans via distinct modes of action. Hum. Mol. Genet. https://doi. org/10.1093/hmg/ddw314 (2016).

93. Brown, A. A. et al. Genetic interactions affecting human gene expression identified by variance association mapping. Elife 3, e01381 (2014).

94. Marshak, D. R., Lukas, T. J. \& Watterson, D. M. Drug-protein interactions: binding of chlorpromazine to calmodulin, calmodulin fragments, and related calcium binding proteins. Biochem.-Us 24, 144-150 (1985).

95. Newman-Tancredi, A. et al. Agonist and antagonist actions of antipsychotic agents at $5-\mathrm{HT}_{1 \mathrm{~A}}$ receptors: a $\left[{ }^{35} \mathrm{~S}\right] \mathrm{GTP} \gamma \mathrm{S}$ binding study. Eur. J. Pharm. 355, 245-256 (1998)

96. Law, C. W., Chen, Y., Shi, W. \& Smyth, G. K. voom: Precision weights unlock linear model analysis tools for RNA-seq read counts. Genome Biol. 15, R29 (2014).

97. Benjamini, Y. \& Hochberg, Y. Controlling the false discovery rate: a practical and powerful approach to multiple testing. J. R. Stat. Soc. Ser. B (Methodol.) 57, 289-300 (1995).

98. Liberzon, A. et al. The Molecular Signatures Database (MSigDB) hallmark gene set collection. Cell Syst. 1, 417-425 (2015).

99. Jia, P., Han, G., Zhao, J., Lu, P. \& Zhao, Z. SZGR 2.0: a one-stop shop of schizophrenia candidate genes. Nucleic Acids Res. 45, D915-D924 (2017).

\section{ACKNOWLEDGEMENTS}

We thank Michael Gandal for sharing the processed microarray data, Barbara Gastel for critically reading and editing the paper, and Ahmad Kawam for helping with method development. We acknowledge the Texas A\&M Institute for Genome Sciences and Society (TIGSS) for providing computational resources. J.J.C. was supported by the TAMU 2019 X-Grants and NIH grant R21Al126219. This work was also supported by the National Natural Science Foundation of China (Nos. 61573296 and 61803320) and the fund of China Scholarship Council to G.H. Brain tissue for the CommonMind Consortium study was obtained from the following brain-bank collections: the Mount Sinai NIH Brain and Tissue Repository, the University of Pennsylvania Alzheimer's Disease Core Center, the University of Pittsburgh NeuroBioBank and Brain and Tissue Repositories, and the NIMH Human Brain Collection Core. CMC Leadership: P. Sklar, J. Buxbaum (Icahn School of Medicine at Mount Sinai), B. Devlin, D. Lewis (University of Pittsburgh), R. Gur, C.-G. Hahn (University of Pennsylvania), K. Hirai, H. Toyoshiba (Takeda Pharmaceuticals Company), E. Domenici, L. Essioux (F Hoffman-La Roche), L. Mangravite, M. Peters (Sage Bionetworks), T. Lehner, B. Lipska (NIMH).

\section{AUTHOR CONTRIBUTIONS}

G.H. and D.O. contributed equally to this paper. G.H., D.O. and J.G. processed and analyzed the data; J.J.C. conceived the presented idea and designed the presented statistical test; G.J. and J.J.C. supervised the project and wrote the paper. All authors discussed the results and contributed to the final paper.

\section{COMPETING INTERESTS}

The authors declare no competing interests.

\section{ADDITIONAL INFORMATION}

Supplementary information is available for this paper at https://doi.org/10.1038/ s41537-020-0097-5.

Correspondence and requests for materials should be addressed to G.J. or J.J.C.

Reprints and permission information is available at http://www.nature.com/ reprints

Publisher's note Springer Nature remains neutral with regard to jurisdictional claims in published maps and institutional affiliations.

Open Access This article is licensed under a Creative Commons Attribution 4.0 International License, which permits use, sharing, adaptation, distribution and reproduction in any medium or format, as long as you give appropriate credit to the original author(s) and the source, provide a link to the Creative Commons license, and indicate if changes were made. The images or other third party material in this article are included in the article's Creative Commons license, unless indicated otherwise in a credit line to the material. If material is not included in the article's Creative Commons license and your intended use is not permitted by statutory regulation or exceeds the permitted use, you will need to obtain permission directly from the copyright holder. To view a copy of this license, visit http://creativecommons. org/licenses/by/4.0/.

(c) The Author(s) 2020 